\title{
THE RELATION BETWEEN LEFT ATRIAL HYPERTENSION AND LYMPHATIC DISTENSION IN LUNG BIOPSIES
}

\author{
BY \\ DONALD HEATH AND PETER HICKEN \\ From the Department of Pathology, University of Birmingham
}

(RECEIVED FOR PUBLICATION SEPTEMBER 2, 1959)

Horizontal lines are frequently seen at the lung bases in radiographs of patients with mitral stenosis (Kerley, 1951). These may be persistent or transient. The persistent lines are due to the deposition of haemosiderin in the fibrous tissue septa between the secondary lobules (Fleischner and Reiner, 1954). The transient lines, seen during periods of cardiac failure, and disappearing with recovery from pulmonary oedema, consist of oedema of the connective tissue of the septa together with distension of the lymphatics (Gough, 1955). Transient basal horizontal lines are accompanied by a raised left atrial blood pressure and invariably appear when the mean pressure in that chamber exceeds $24 \mathrm{~mm}$. $\mathrm{Hg}$, but elevation of pulmonary arterial pressure alone appears to play little part in the production of the lines (Rossall and Gunning, 1956). The present investigation was performed to see if this relation could be demonstrated by direct histological observation of the size of pulmonary lymphatic vessels in biopsies of the lingula pulmonis in patients in whom the left atrial or pulmonary "arterial wedge" pressure had been measured. If pulmonary lymphatic distension could be related to pulmonary venous hypertension by this technique, it would a valuable aid to the morbid anatomist in the interpretation of structural changes in lung biopsies in terms of haemodynamics.

\section{Method AND Material}

Clinical Material.-Twenty cases of mitral stenosis of rheumatic aetiology were studied. These were patients at the City General Hospital, Sheffield, under the medical care of Dr. W. Whitaker (Table). There

TABLE

DEGREE OF PULMONARY LYMPHATIC DISTENSION AND BLOOD PRESSURES IN PULMONARY CIRCULATION AND LEFT ATRIUM IN 20 CASES OF MITRAL STENOSIS

\begin{tabular}{|c|c|c|c|c|c|c|c|}
\hline \multirow{2}{*}{$\begin{array}{l}\text { Case } \\
\text { No.* }\end{array}$} & \multirow{2}{*}{$\underset{\text { (Years) }}{\text { Age }}$} & \multirow{2}{*}{$\begin{array}{l}\text { Pulmonary } \\
\text { Artery Mean } \\
\text { Blood Pressure } \\
(\mathrm{mm} . \mathrm{Hg})\end{array}$} & \multirow{2}{*}{\begin{tabular}{|c|} 
Pulmonary \\
“ Arterial Wedge” \\
or Left Atrial Blood \\
Pressure (mm. Hg)
\end{tabular}} & \multicolumn{3}{|c|}{ Grade of Lymphatic Distension } & \multirow{2}{*}{$\begin{array}{c}\text { Incidence of } \\
\text { Lymphangiec- } \\
\text { tasis } †\end{array}$} \\
\hline & & & & In Pleura & $\begin{array}{l}\text { In Interlobular } \\
\text { Fissures }\end{array}$ & $\begin{array}{c}\text { Around Pul- } \\
\text { monary Arteries }\end{array}$ & \\
\hline
\end{tabular}

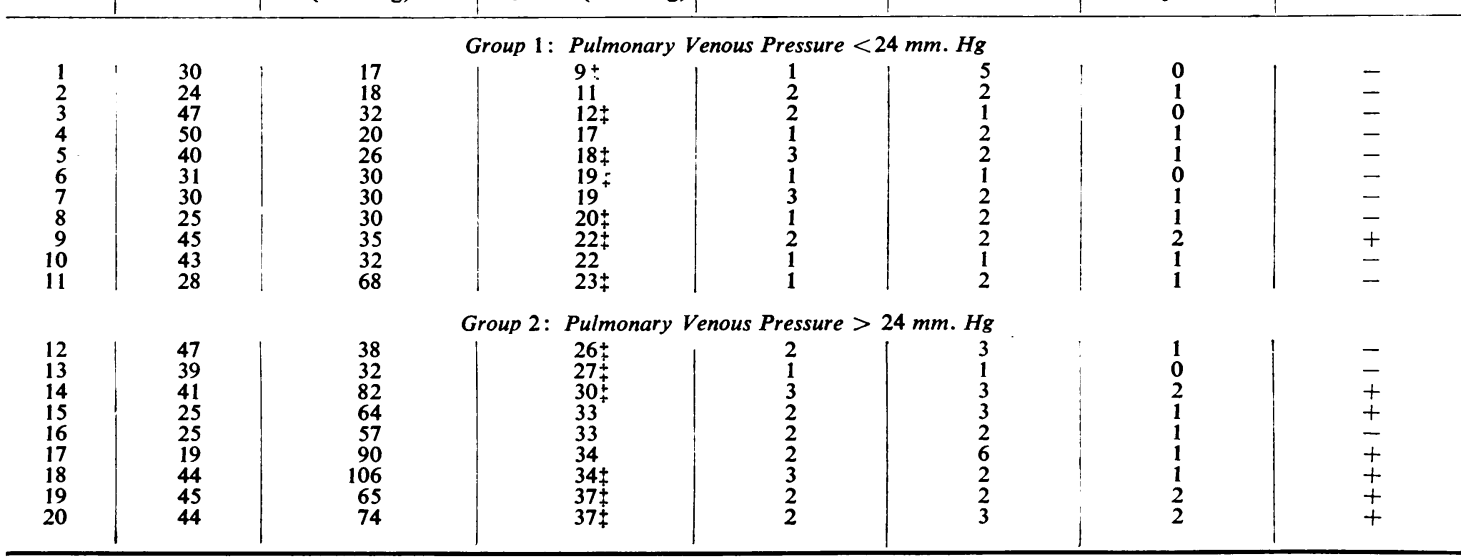

* Cases are arranged in order of ascending pulmonary venous mean pressure. $\dagger=$ See text for definitions. $\ddagger=$ Left atrial blood pressure. 
were 19 women and one man (Case 10), and their ages ranged from 19 to 50 years. These included seven cases in which the pulmonary artery blood pressure and the pulmonary " artery wedge" pressure were measured at cardiac catheterization by one of us (D.H.) as described below. The pulmonary artery blood pressure and the left atrial blood pressure were measured in the other 13 cases by the bronchoscopic method of Allison and Linden (1953) by Professor P. R. Allison at the General Infirmary, Leeds. The pulmonary arterial pressure ranged from 17 to $106 \mathrm{~mm}$. Hg. The left atrial or pulmonary "arterial wedge" pressure was considered to be equal to the "pulmonary venous blood pressure" which therefore ranged from 9 to $37 \mathrm{~mm}$. $\mathrm{Hg}$. These cases were considered in two groups according to the level of the "pulmonary venous blood pressure." Group I comprises those in which this pressure was less than, and Group II those in which it was greater than, $24 \mathrm{~mm}$. $\mathrm{Hg}$, this pressure having been found by Rossall and Gunning (1956) to be critical for the appearance of transient basal horizontal lines on radiographs.

Measurement of Blood Pressures in Pulmonary Circulation and Left Atrium.-A cardiac catheter was introduced, under fluoroscopic control, into the right atrium through a systemic vein in the forearm. The tip of the catheter was advanced through the tricuspid valve, right ventricle, and pulmonary valve until it came to lie in the right or left pulmonary artery. The pressure in the pulmonary artery was then measured by a saline manometer, and the systolic, diastolic, and mean blood pressures were recorded by a condenser manometer. The tip of the catheter was then advanced until it wedged in a small pulmonary artery. In this position the pressure fell below that in the main branches of the pulmonary artery, and when settled was assumed to record the pulmonary venous blood pressure, since it has been shown by Epps and Adler (1953) that the arterial "wedge pressure" or pulmonary-capillary blood pressure is identical with that in the left atrium. The cardiac catheter was assumed to be in a satisfactory position for recording the pulmonary venous pressure when the pulse record was venous in character and when a blood sample taken through the catheter had an oxygen saturation similar to that in the systemic arterial blood.

Histological Material. - Lung tissue was obtained for examination by biopsy at the time of thoracotomy by Professor P. R. Allison at Leeds General Infirmary and Mr. J. T. Chesterman at the City General Hospital, Sheffield. The lingula was inflated and a piece of lung tissue, about $2 \mathrm{~cm}$. across and 0.5 to $1.0 \mathrm{~cm}$. thick, was resected after either two Pott's clamps or suture material had been inserted across it. This tissue was fixed in formolsaline. Paraffin sections were stained routinely with haematoxylin and eosin, and with Verhoeff's and Van Gieson's stains to demonstrate elastic, fibrous, and muscular tissue.
Histological Structure of Lymphatics.-The pulmonary lymphatics were identified on the criteria of Miller (1947). The walls of the larger lymphatics consist of collagen, small fragmented elastic fibrils, and smooth muscle fibres which are longitudinally and obliquely orientated; they are lined by endothelium (Fig. 1). The muscle in the walls of the lymphatics is arranged focally and does not form a continuous muscular wall as in pulmonary arteries and veins. Hence much of the wall is lined only by endothelium even when the lymphatic vessel exceeds $100 \mu$ in diameter (Fig. 1). Pulmonary lymphatics

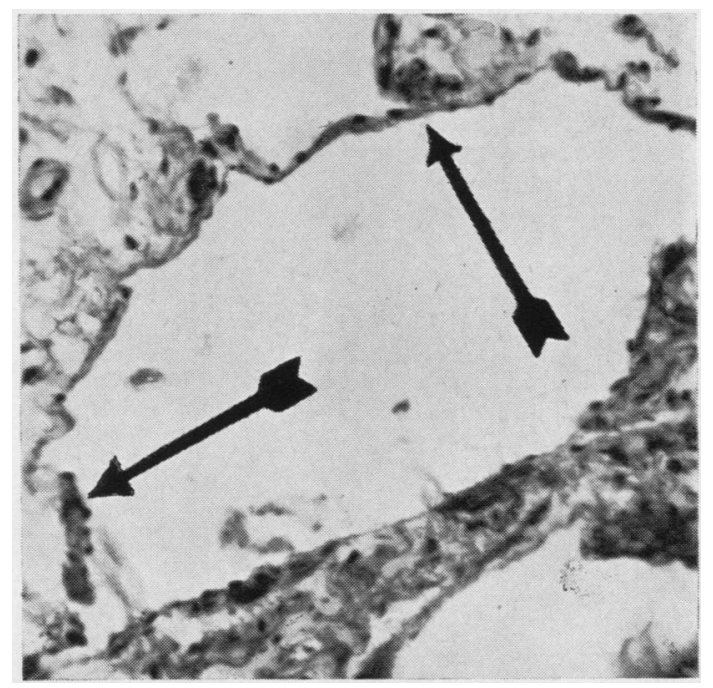

FIG. 1.-Case 14. Oblique section of a large pulmonary lymphatic vessel showing two focally arranged groups of smooth muscle fibres (indicated by black arrows). A valve is situated between the lymphatic trunk and one of its tributaries. $(\times 215$.)

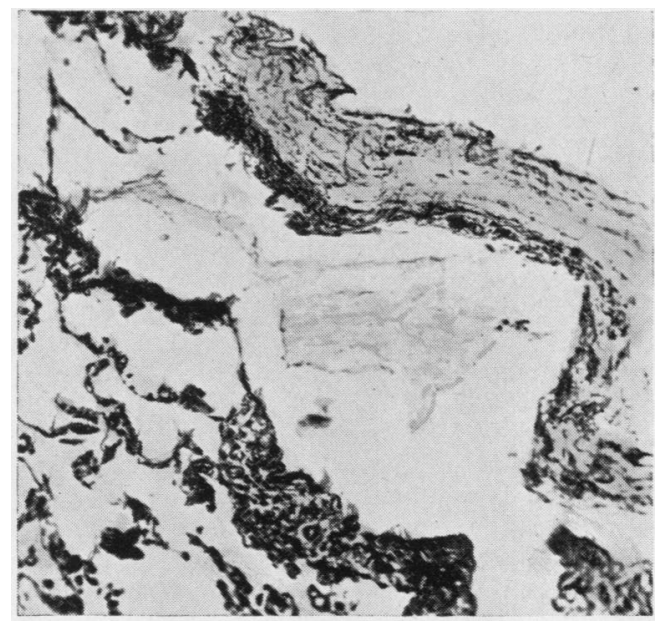

Fig. 2.-Case 14. Pleural lymphatic vessel showing great variation in diameter over a short distance. $A$ valve and lymphatic capillaries are present. $(\times 125$. $)$ 


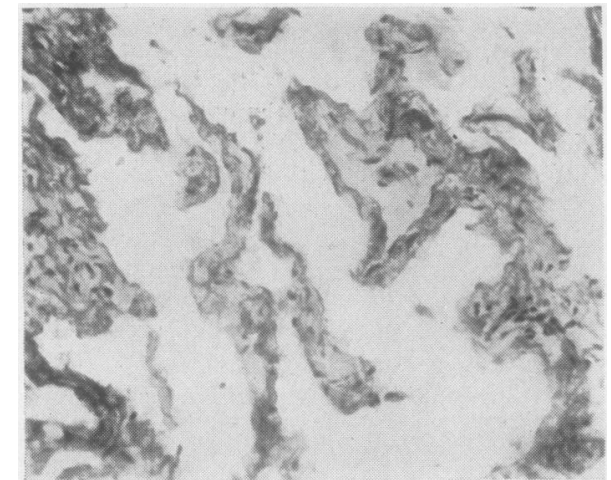

FIG. 3.-Case 14. Lymphatic vessels and capillaries in the connective tissue septa between secondary lobules. These have an endothelial lining without muscle in their walls. Contrast with the structure of the larger lymphat ic vessels shown in Fig. $1 .(\times 150$.

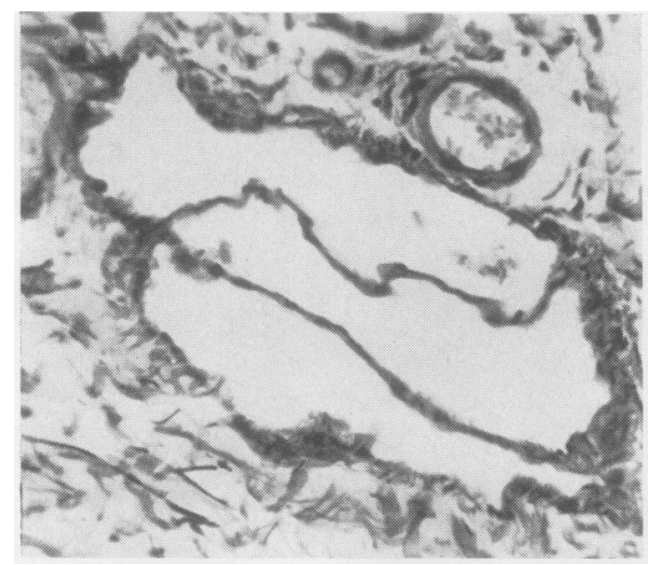

FIG. 4.-Case 16. Pleural lymphatic vessel showing a pair of valves. ( 250.)

differ from veins in that their diameter varies greatly within short distances, suddenly narrowing and then immediately expanding into wide segments (Fig. 2). They do not show the intimal fibrosis which is so characteristic of the veins of the adult human lung. The quantity of elastic tissue in the wall is also less. The smaller lymphatics comprise a fibro-elastic coat and an endothelial lining and the lymph capillaries consist solely of endothelium (Fig. 3). Valves are common in the pulmonary lymphatics, especially in the pleura (Fig. 4). These are usually arranged in pairs, are formed of intimal folds supported by a minimal amount of fibrous tissue, and aid the ready identification of lymphatics (Figs. 2 and 4).

The lymphatics of the human lung are arranged in superficial and deep networks. The former occur in the pleura (Fig. 2). The latter are situated around the bronchi, pulmonary arteries (Fig. 5), and veins and in the connective tissue septa between the secondary lobules (Fig. 3). The two systems anasto- mose in the pleura and at the hilum. Miller (1947) has demonstrated by injection studies that the superficial lymphatic vessels form a pattern in the pleura of irregular polyhedra which mark out the secondary lobules. Smaller lymphatics, with finger-like projections, delineate smaller zones within the lobular area. $\mathrm{He}$ has shown that only one or two lymphatics accompany the smaller pulmonary veins but that there is a network around the larger veins; this communicates with the bronchial network at points where pulmonary venous radicles arise from the bronchial tree. Two or three main lymph trunks accompany each pulmonary artery and loops from these form a network around the vessel; the trunk situated between bronchus and pulmonary artery is common to both networks.

Assessment of Degree of Lymphatic Distension.-Two methods were employed in each case.

(1) The diameters of the largest single lymphatic seen in the pleura (Fig. 2), in the connective tissue septa between secondary lobules including those around veins (Fig. 3), and around pulmonary arteries (Fig. 5) were measured and graded according to the following system.

Grade 0: No lymphatics seen

Grade 1: Largest lymphatic seen was not more than $100 \mu$ in diameter

Grade 2: Largest lymphatic seen was between 100 and 200 ": in diameter

Grade 3: Largest lymphatic seen was between 200 and $300 \%$ in diameter

Grade 4: Largest lymphatic seen was between 300 and $400 \mu$ in diameter; and so on.

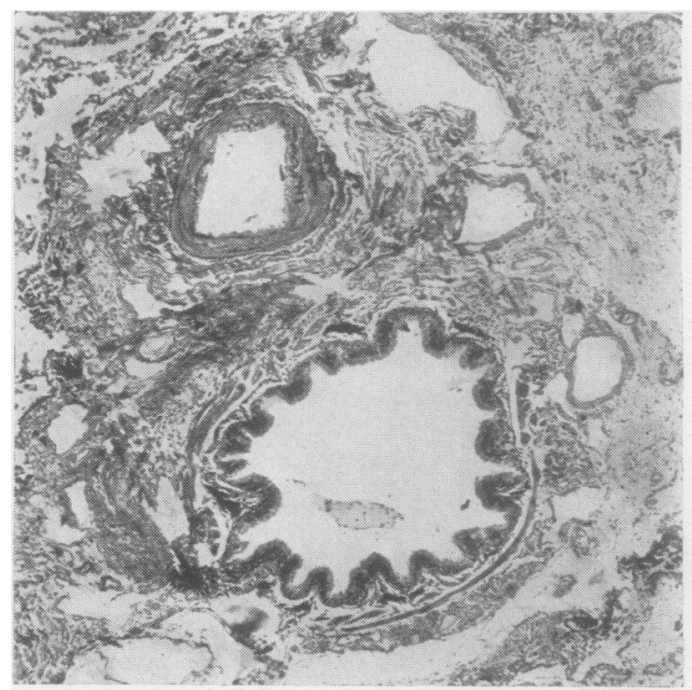

FIG. 5.-Case 14. Transverse section of a pulmonary artery and bronchus with surrounding distended lymphatic vessels. The appearances are those of pulmonary lymphangiectasis as defined in the text. ( $\times 50$.)

All sections were stained with Verhoeff's and Van Gieson's stains. 
(2) The presence of many distended lymphatics around pulmonary arteries was regarded as " pulmonary lymphangiectasis" (Fig. 5). When the lymphatics in this site were few and less than $100 \mu$ in diameter, as in the normal lung, it was considered that lymphangiectasis was absent. An attempt at objectivity was made in that one of us (P.H.) made these observations without prior knowledge of the haemodynamic data. The results are shown in the Table.

\section{Discussion}

The aim of this investigation was to determine if the pathologist can detect the presence of pulmonary venous hypertension from the appearance of lymphatic distension in lung biopsies. Two methods of assessment of lymphatic distension were employed, depending in the one instance upon the diameter of the largest single lymphatic seen in each of the three specified sites, and in the other instance upon the overall appearance of large numbers of distended lymphatics (Fig. 5). It is important to know which of these two methods is reliable for future use, and so the first consideration is to see in which of the two methods the already proven relation between left atrial (pulmonary venous) hypertension and lymphatic distension is more clearly demonstrated.

Employing the first method, we found no relation between the degree of lymphatic distension and the level of pulmonary venous blood pressure. For instance, in Case 1, grade 5 pulmonary lymphatics were seen in the connective tissue septa between the secondary lobules when the pulmonary venous blood pressure was only $9 \mathrm{~mm}$. Hg. However, only grade 1 dilatation was found in the same site in Case 13, where there was a pulmonary venous hypertension of $27 \mathrm{~mm}$. $\mathrm{Hg}$. Indeed, the data in the Table demonstrate the complete lack of relation between the diameter of the largest lymphatic in any of the three specified sites and the magnitude of the pulmonary " arterial wedge" or left atrial blood pressures. The range of gradings observed in the three sites in groups 1 and 2 are almost identical when compared: Group 1: Pleura, grades 1 to 3 ; interlobular fissures, grades 1 to 5 ; around pulmonary arteries, grades 0 to 2 . Group 2: Pleura, grades 1 to 3 ; interlobular fissures, grades 1 to 6 : around pulmonary arteries, grades 0 to 2 . The reason for this lack of relation is obvious to those familiar with the microscopical structure of pulmonary lymphatics which, as demonstrated by Miller (1947), differ from pulmonary arteries and veins in that their diameter varies greatly within short distances, suddenly narrowing and then immediately expanding into wide segments (Fig. 2). Hence the mensuration of individual pulmonary lymphatics is valueless for the present purpose in contrast to the application of this technique to pulmonary arteries, which has been extensively used for the measurement of lumen-to-wall ratios by Dammann and Ferencz (1956).

The second method of assessing the degree of distension of pulmonary lymphatics, depending on the overall appearance of these vessels around pulmonary arteries, proves to be more valid. "Pulmonary lymphangiectasis," as previously defined (Fig. 5), is detected easily and was present in six of the seven cases with a pulmonary venous blood pressure of $30 \mathrm{~mm}$. $\mathrm{Hg}$ or more, and in only one of the 13 cases where the corresponding pressure was below that figure (Case 9, $22 \mathrm{~mm}$. $\mathrm{Hg}$ ).

Rossall and Gunning (1956) found that transient basal horizontal lines appeared on the radiographs of patients with mitral stenosis when the left atrial blood pressure exceeded $24 \mathrm{~mm}$. Hg. Comparing our results with theirs it is interesting to note that histological evidence of lymphatic distension appears when the pulmonary venous blood pressure is raised above $30 \mathrm{~mm}$. $\mathrm{Hg}$ and an associated pulmonary arterial hypertension exceeding $60 \mathrm{~mm}$. Hg. This relation is not invariable, since in Case 9 pulmonary lymphangiectasis appeared with only moderate elevation of the blood pressure in the pulmonary arteries and veins. This observation is of interest when one recalls that pulmonary haemosiderosis has been shown to have the same type of relation to co-existent pulmonary arterial and venous hypertension (Heath and Whitaker, 1956).

In the pulmonary lymphatics there are no structural changes comparable to those which occur in pulmonary arteries and veins subjected to hypertension. It should be noted that in none of the cases studied was there evidence of pulmonary infection, which might itself give rise to lymphatic distension. Clearly the present technique cannot be employed when there are potent causes for lymphatic distension other than haemodynamic ones.

In conclusion, we believe that the second method of histological assessment of pulmonary lymphatic distension is reliable in detecting the presence of severe pulmonary venous hypertension in lung biopsies.

\section{SUMMARY}

The degree of distension of the pulmonary lymphatics was assessed in lung biopsies resected 
from 20 patients with mitral stenosis in whom the pulmonary artery and "pulmonary venous" (pulmonary " arterial wedge" or left atrial) blood pressures were known. Two methods of assessment of lymphatic distension were employed, depending in the one instance upon the diameter of the largest single lymphatic seen in each of three specified sites, and in the other instance upon the overall appearance of distended lymphatics around pulmonary arteries. The measurement of the diameter of individual lymphatics proved to be valueless.

Pulmonary lymphangiectasis, as assessed by the second method, was observed in seven cases and in six of these the pulmonary venous blood pressure was $30 \mathrm{~mm}$. $\mathrm{Hg}$ or more.

It is concluded that there is a relation between severe pulmonary venous hypertension and lymphatic distension visible as lymphangiectasis around pulmonary arteries as seen in lung biopsies.

\section{REFERENCES}

Allison, P. R., and Linden, R. J. (1953). Circulation, 7, 669.

Dammann, J. F., Jr., and Ferencz, C. (1956). Amer. Heart J., 52, 7. Epps, R. G., and Adler, R. H. (1953). Brit. Heart J., 15, 298.

Fleischner, F. G., and Reiner, L. (1954). New Engl. J. Med., 250, 900. Gough. J.(1955), Lancet, 1, 161.

Heath, D., and Whitaker, W. (1956), J. Path. Bact., 72, 531.

Heath, D., and Whitaker, W. (1956), J. Path. Bact., 72, 531. S. C. Shanks and P. Kerley, Vol. 2. Lewis, London.

Miller, W. S. (1947). The Lung, 2nd ed. Thomas,'Springfield, Illinois Rossall, R. E., and Gunning, A. J. (1956). Lancet, 1, 604. 